九州大学学術情報リポジトリ

Kyushu University Institutional Repository

\title{
GENERALISED MEASURE OF DISPERSION AND ANALYSIS OF DISTRIBUTION PATTERN
}

Kitagawa, Toshio

Kyushu University

https://doi.org/10.5109/13031

出版情報: 統計数理研究. 13 (1/2)，pp.53-71，1968-03. Research Association of Statistical Sciences

バージョン：

権利関係 : 


\title{
GENERALISED MEASURE OF DISPERSION AND ANALYSIS OF DISTRIBUTIONAL PATTERN ${ }^{1}$
}

\author{
By \\ Tosio Kitagawa \\ (Received December 25, 1967)
}

\section{$\S 1$. Introduction.}

For measuring dispersion of distribution of numbers of individuals in spatial area and analysing their distributional pattern, a certain index of dispersion has been advocated and used by many research workers since Simpscn [6] including ecologists and entomologists, and its theoretical aspects have been deeply discussed by an animal ecologist, Morishita [2], [3], [4] and [5]. The author of the present paper has been for these several years in close contact with Morishita in this research area of the problems, and it is the purpose of the present paper to explore certain mathematical principles, which can secure us to establish a unified standpoint in dealing with a generalised measure of dispersion, and which, at the same time, may provide us a certain set of tools as substantial techniques in getting penetrated observations on distributional pattern.

In section 2 we shall introduce three basic notions upon which our formulation of $\phi$-dispersions will be established: (1) multistage decomposition system $D^{(m)}(-1)$ of the set $A$; (2) $x$-variates associated with $D^{(m)}(A)$; (3) $d$-depth $\phi$-dispersion of a function $f$ on the set $A_{i_{1} i_{2} \cdots i_{h}}$ with respect to $D^{(d)}\left(A_{i_{1} i_{2} \cdots i_{h}}\right)$ where $A_{i_{1} i_{2} \cdots i_{h}}$ is a set belonging to it. In section 3 another essential set-up is introduced which is called as distributional pattern with one parameter generalised exponential type distributions associated with the decomposition $D^{(m)}(A)$. There are also two important notions, homogenity and inheritance, in this connection. A few examples given in section 3 will serve to show that important special cases discussed by Morishita [1] and Smith [5] can be explained in term of these notions in our present formulations, including binomial, non-negative and Poisson distributions as our special examples. The main purpose of this paper is then to evalute the conditional expectation of various $\phi$-dispersions.

For this purpose there is a crucial property of the concurrence function $\phi$ with respect to the assigned additive family of generalised exponential type distributions, which we shall define in section 4 as being reproductive. After these preparations we are now in the position to give the conditional expectation of $\phi$-dispersion for such a reproductive $\phi$-function. The main result in this section is enunciated in Proposition 6 of which Propositions 4 and 5 are special cases. They show that these conditional expectations of $\phi$-dispersion can be obtained in concise forms. For instance

1) This work was done by the grant Tokutei-Kenkyu of the Ministry of Education for the PT (Productivity of Terrestorial Ecosystem) Section of the International Biological Project. 
Proposition 3 gives the simple formula (j.1) for the 1-depth $\phi$-dispersion in the set $A$.

In section 6 we discuss partitional pattern of additive parameters in the decomposition system $D^{(m)}(A)$ with particular reference to two extreme cases, equipartition and monopolistic concentration. In section 7 we turn to discuss $\phi$-dispersion as function of size variable associated with the decomposition system $D^{(m)}(A)$. An interpolation procedure for getting size variable to penetrate into finer and global structures is explained in virtue of the notions of homogenity and inheritance previously defined. Section 8 is devoted to explain the relations between our $\phi$-dispersion and current index of dispersion introduced and discussed by Morishita $[2] \sim[5]$. We shall show that the latter is a particular example of the former apart from a certain multiplier which is suited for Poisson distribution. Two stage and three stage distributional patterns are illustrated in order to show the uses of $\phi$-dispersions as functions of size variables with particular reference to binomial, negative binomial and Poisson distributions.

These results which are summarized briefly here may be said to give us some tools for theoretical analysis of various empirical results obtained by ecologists for natural and artificial populations. On the other hand any sampling theory regarding the uses of our $\phi$-dispersions is not discussed in this paper, and we expect to have another occasion to develop it.

It is also noted that the formulation of the present paper is partly due to that of additive family of exponential type which was discussed by the author [1], whose detailed results are not required for the understanding of the present paper.

\section{$\S 2 . \quad \phi$-dispersion in multistage distributional pattern.}

For introducing a notion of $\dot{\phi}$-dispersions regarding $x$-variate in a set $A$, there are three fundamental notions upon which it can be established.

(1) Multistage decomposition system $D^{(m)}(A)$ of the set $A$. $A$ set $A$ is $\operatorname{decomposed}$ into the sum of $q_{1} q_{2} \cdots q_{k}$ mutually disjoint subsets $A_{i_{1} i_{2} \cdots i_{m}}\left(i_{j}=1,2, \cdots, q_{j} ; j=1,2, \cdots, m\right)$ in such a way that

$\left(1^{\circ}\right) \quad A=\sum_{i_{1}=1}^{q_{1}} A_{i_{1}}$

$\left(2^{\circ}\right) \quad A_{i_{1} i_{2} \cdots i_{h}}=\sum_{i_{h+1}=1}^{q_{h+1}} A_{i_{1} i_{2} \cdots i_{h} i_{h+1}}, \quad\left(i_{j}=1,2, \cdots, q_{j} ; j=1,2, \cdots, h\right)$

for $h=1,2,3, \cdots, m-1$.

The set $\left\{A_{i_{1} i_{2} \cdots i_{m}}\right\}$ is said to constitute the (m-stage) decomposition system $D^{(m)}(A)$ of the set $A$.

The family of $q_{1}$ subsets enunciated in $\left(1^{\circ}\right)$ and those of $q_{1} q_{2} \cdots q_{h}$ subsets enunciaied in $\left(2^{\circ}\right)$ are said to constitute the first stage decomposition $D_{1}^{(m)}(A)$ and the $(h+1)$-th stage decomposition $D_{h-1}^{(m)}(A)(h=1,2, \cdots, m-1)$ respectively. Under $m$-stage decomposition system $D^{(m)}(A)$ there are imbedded the lower stage decomposition systems $D^{(n)}(A)$ for $n=1,2, \cdots, m-1$, and it is immediate to observe that, for each subset $A_{i_{1} i_{2} \cdots i_{h}}$, we can define the $l$-stage decomposition system $D^{(l)}\left(A_{i_{1} i_{2} \cdots i_{h}}\right)$ and its $\nu$-th stage decomposition $D_{\nu}^{l}\left(A_{i_{1} i_{2} \cdots i_{h}}\right)$ for $\nu=1,2, \cdots, l ; l=1,2, \cdots, m-h$. In view of these facts, for the sake of convenience, we may and we shall consider the set $A$ as 
the unique subset of the set $A$ at the zero-th stage decomposition, say, $D_{0}^{(m)}(A)$, and consider it as an element of the family of the sets $\left\{A_{i_{\perp} i_{2} \cdots i_{h}}\right\}$ corresponding to the case $h=0$.

(2) $x$-variates associated with an m-stage decomposition system $D^{(m)}(A)$. A set of $q_{1} q_{2} \cdots q_{m}$ non-negative integers $\left\{x_{i_{1} i_{2} \cdots i_{m}}\right\}\left(i_{j}=1,2, \cdots, q_{j} ; j=1,2, \cdots, m\right)$ is assumed to be assigned, and the following set of $x$-variates is defined in correspondence with the decomposition system $D^{(m)}(A)$ :

(1 $\left.1^{\circ}\right) \quad x=\sum_{i_{1}=1}^{q_{1}} x_{i_{1}}$

$\left(2^{\circ}\right) \quad x_{i_{1} i_{2} \cdots i_{h}}=\sum_{i_{h+1}=1}^{q_{h+1}} x_{i_{1} i_{2} \cdots i_{h} i_{h+1}}, \quad\left(i_{j}=1,2, \cdots, q_{j} ; j=1,2, \cdots, h\right)$

for $h=1,2, \cdots, m-1$.

(3) $\phi$-dispersions. A function $f(x)$ is assumed to be defined in the set $\mathfrak{D}_{f}$ which is a subset of the set of all non-negative integers. Among such functions let us choose a function $\dot{\phi}$ with whose reference we introduce

Definition 1. A d-depth $\phi$-measure of dispersion, abbreviated by $\phi$-dispersion, of a function $f$ on the set $A_{i_{1} i_{2} \cdots i_{h}}$ with respect to the decomposition system $D^{(d)}\left(A_{i_{1} i_{2} \cdots i_{h}}\right)$ is defined as

$$
\phi_{d}(f)\left(A_{i_{1} i_{2} \cdots i_{h}}\right) \equiv \frac{1}{\phi\left(x_{i_{1} i_{2} \cdots i_{h}}\right)} \sum_{i_{h+1}=1}^{q_{h+1}} \sum_{i_{h+2}=1}^{q_{h+2}} \cdots \sum_{i_{h+d}=1}^{q_{h+d}} \phi\left(x_{i_{1} \cdots i_{h} i_{h+1} \cdots i_{h+d}}\right) f\left(x_{i_{1} \cdots i_{h+d}}\right),
$$

for every $i_{j}=1,2, \cdots, q_{j} ; j=1,2, \cdots, h ; h=0,1,2, \cdots, m-1$.

Particularly when $f(x)$ becomes to be identical with a value 1 (identity) over the domain $\mathfrak{D}_{f}$, we shall write

$$
\phi_{d}\left[A_{i_{1} i_{2} \cdots i h}\right]=\phi_{d}(1)\left(A_{i_{1} i_{2} \cdots i h}\right) .
$$

As a direct consequence of Definition 1, we have

Proposition 1. We have

$$
\phi_{d_{1}}\left(\phi_{d_{2}}(f)\right)\left(A_{i_{1} i_{2} \cdots i_{h}}\right)=\phi_{d_{1}+d_{2}}(f)\left(A_{i_{1} i_{2} \cdots i_{h}}\right) .
$$

Among various $\phi$-dispersion of a function $f$ there are specific important cases which are of our particular interests. These occur when at least one of the following three conditions is satisfied: (i) $A_{i_{1} i_{2} \cdots i_{h}}=A$, that is, the case when $h=0$ formally ; (ii) the function $f(x)$ is identical with 1 for every $x$ in $\mathscr{D}_{f}$; (iii) the depth $d$ is equal to 1 . For illustration a few examples are given.

Example 2.1. The $d$-depth dispersion of identity

and

$$
\phi_{d}(1)(A)=\phi_{d}[A]=-\frac{1}{\phi(x)} \sum_{i_{1}=1}^{q_{1}} \sum_{i_{2}=1}^{q_{2}} \cdots \sum_{i_{d}=1}^{q_{d}} \phi\left(x_{i_{1} i_{2} \cdots i_{d}}\right)
$$

$$
\phi_{d}(1)\left(A_{i_{1} i_{2} \cdots i_{h}}\right)=\phi_{d}\left[A_{i_{1} i_{2} \cdots i_{h}}\right]=\frac{1}{\phi\left(x_{i_{1} i_{2} \cdots i_{h}}\right)} \sum_{i_{h+1}=1}^{q_{h+1}} \ldots \sum_{i_{h}+d=1}^{q_{h}+d} \phi\left(x_{i_{1} \cdots i_{h} i_{h+1} \cdots i_{h+d}}\right) .
$$

Example 2.2. The 1-depth dispersion of function $f$

$$
\begin{gathered}
\phi_{1}(f)(A)=\frac{1}{\phi(x)} \sum_{i_{1}=1}^{q_{1}} \phi\left(x_{i_{1}}\right) f\left(x_{i_{1}}\right), \\
\phi_{1}(f)\left(A_{i_{1} i_{2} \cdots i_{h}}\right)=\frac{1}{\phi\left(x_{i_{1} \cdots i_{h}}\right)} \sum_{i_{h+1}=1}^{q_{h+1}} \phi\left(x_{i_{1} \cdots i_{h} i_{h+1}}\right) f\left(x_{i_{1} \cdots i_{h} i_{h} \div 1}\right) .
\end{gathered}
$$


Example 2.3. There are a lot of possibilities in choosing the basic function $\dot{\phi}$ in defining our $\phi$-dispersion (2.1). The following particular examples are important:

$$
\phi^{[j]}(x)=x(x-1) \cdots(x-j+1)=x^{[i]}, \quad \mathfrak{D} \phi^{[j]}=[x ; x \geqq j]
$$

for $j \geqq 2$. Indeed the current index of dispersion discussed by Morishita [2] [4] is in our notation

$$
I_{\grave{o}}=q_{1} \phi_{\overline{1}}^{[2]}[A]=\frac{q_{1}}{\phi_{1}^{23}(x)} \sum_{i_{1}=1}^{q_{1}} \phi\left(x_{i_{1}}\right)=I_{1}^{2-2}[A] .
$$

In a more general aspect a $d$-depth $[j]$-index of dispersion of the function $f$ on the set $A_{i_{1} i_{2} \cdots i_{n}}$ is defined by

$$
I_{d}^{j j}(f)\left(A_{i_{1} i_{2} \cdots i_{h}}\right)=\left(q_{h+1} q_{h+2} \cdots q_{h+d}\right)^{j-1} \phi_{d}^{[j-}\left(A_{i_{1} i_{2} \cdots i_{h}}\right)
$$

in virtue of (2.1). In particular when the function $f$ becomes an identity, (2.10) becomes

$$
\begin{aligned}
I_{\vec{d}}^{[j]}(1)\left(A_{i_{1} i_{2} \cdots i_{h}}\right) & =I_{d}^{[j]}\left[A_{i_{1} i_{2} \cdots i_{h}}\right] \\
& =\frac{\left(q_{h+1} q_{h+2} \cdots q_{h+d}\right)^{j-1}}{x_{i 1 i_{2} \cdots i_{h}}^{[j]}} \sum_{i_{h+1}}^{q_{h+1}} \cdots \sum_{i_{h+d}=1}^{q_{h+d}} x_{i_{1 i 2} \cdots i_{h} i_{h+1} \cdots i_{h+d}}^{[j]} .
\end{aligned}
$$

Multistage distributional patterns have been discussed by some ecologists. In fact, Morishita [2] for instance, discussed intraclump distribution in analysis of distributional patterns by $I_{\hat{o}}$. In this connection it seems to us to be indispensable to introduce a multistage decomposition system $\left\{A_{i_{1} i_{2} \cdots i_{m}}\right\}$, a set of $x$-variates $\left\{x_{i_{1} i_{2} \cdots i_{m}}\right\}$, as we have just done, in order to prepare a mathematical tool by which to explore a multistage distributional pattern.

In some instances which follow, when any confusion may not occur but convenience can be expected to make clear multistage structure, we may use the notation $x_{i_{1} i_{2} \cdots i_{h} \ldots}$ and $x \ldots$ in the place of $x_{i_{1} i_{2} \cdots i_{h}}$ and $x$ respectively. At the same time we may and we shall simplify our notation so as to concentrate our discussion in lower stage decompositions associated with our original decomposition $D^{(m)}(A)$. Thus we may and we shall denote sometimes $x$ and $q$ instead of $x \cdots$ and $q_{1}$ originally defined in the system $D^{(m)}(A)$, when we are particularly concerned with the first stage decomposition.

\section{§3. Distributional pattern with one parameter generalised exponential type distributions associated with the $m$-stage decomposition system $D^{(m)}(A)$ of the set $A$.}

We start with

Definition 2. A set of $q_{1} q_{2} \cdots q_{k}$ stochastic variables $\left\{X_{i_{1} i_{2} \cdots i_{m}}\right\}\left(i_{h}=1,2, \cdots q_{h}\right.$, ; $h=1,2, \cdots, m)$ is assumed to satisfy the following conditions:

(1) Each stochastic variable $X_{i_{1} i_{2} \cdots i_{m}}$ is associated with the set $A_{i_{1} i_{2} \cdots i_{m}}$.

(2) The $q_{1} q_{2} \cdots q_{m}$ stochastic variables $\left\{X_{i_{1} i_{2} \cdots i_{m}}\right\}$ are mutually independent.

(3) The probability density function of $X_{i_{1} i_{2} \cdots i_{m}}$ belongs to the same family of $e x$ ponential type distributions with the probability element

$$
\begin{aligned}
g(x ; & \left.k_{i_{1} i_{2} \cdot i_{m}} \mid A_{i_{1} i_{2} \cdots i_{m}}\right) d \mu_{i_{1} i_{2} \cdots i_{m}}(x) \\
& \equiv \exp \left\{\lambda x+k_{i_{1} i_{2} \cdots i_{m}} b(\lambda)\right\} a_{i_{1} i_{2} \cdots i_{m}}(x) d \mu_{k_{i_{1} i 2 \cdots i_{m}}}(x)
\end{aligned}
$$


with the additive parameter $k_{i_{1} i_{2} \cdots i_{m}}$, which will be denoted briefly by $g\left(x ; k_{i_{1} i_{2} \cdots i_{m}} \mid A_{i_{1} i_{2} \cdots i_{m}}\right)$, where $\mu_{k_{i 1 i 2 \cdots i m}}(x)$ is a step function having jump solely at integral values of $x$.

(4) The set of stochastic variables $\left\{X_{i_{1} i_{2} \cdots i_{h}}\right\}(h=1,2, \cdots, m-1)$ and $X$ is defined in the following way:

$\left(1^{\circ}\right) \quad X=\sum_{i_{1}=1}^{q_{1}} X_{i_{1}}$

$\left(2^{\circ}\right) \quad X_{i_{1} i_{2} \cdots i_{h}}=\sum_{i_{h+1}=1}^{q_{h+1}} X_{i_{1} i_{2} \cdots i_{h} i_{h+1}}, \quad\left(i_{j}=1,2, \cdots, q_{j} ; j=1,2, \cdots, h\right)$

for $h=1,2,3, \cdots, m-1$.

(5) The set of additive constants $\left\{k_{i_{1} i q \cdots i_{h}}\right\}\left(i_{j}=1,2, \cdots, q_{j} ; j=1,2, \cdots, h ; h=1,2\right.$, $\cdots, m-1)$ and $k$ is defined in the following way:

$\left(1^{\circ}\right) \quad k=\sum_{i_{1}=1}^{q_{1}} k_{i_{1}}$

$\left(2^{\circ}\right) \quad k_{i_{1} i_{2} \cdots i_{h}}=\sum_{i_{h+1}=1}^{q_{h+1}} k_{i_{1} i_{2} \cdots i_{h} i_{h+1}}, \quad\left(i_{j}=1,2, \cdots, q_{j} ; j=1,2, \cdots, h\right)$

for $h=1,2,3, \cdots, m-1$.

Then the set of $q_{1} q_{2} \cdots q_{m}$ stochastic variables $\left\{X_{i_{1} i_{2} \cdots i_{h}}\right\}$ is said to constitute an additive family of one parameter exponential type distributions $\left\{g\left(x ; k_{i_{1} i_{2} \cdots i_{m}} \mid A_{i_{1} i_{2} \cdots i_{m}}\right)\right\}$ associated with the m-stage decomposition system $D^{(m)}(A)$ of the set $A$, and it is denoted by

$$
\left\{X_{i_{1} i_{2} \cdots i_{m}}\right\} \in E_{\lambda}\left(k_{i_{1} i_{2} \cdots i_{m}} ; D^{(m)}(A)\right) .
$$

Now it is immediate to observe

Proposition 2. Under the assumption (3.2), the set of $q_{1} q_{2} \cdots q_{h}$ stochastic variables $\left\{X_{i_{1} i_{2} \cdots i_{h}}\right\}$ constitutes an additive family of one parameter exponential type distributions $\left\{g\left(x ; k_{i_{1} i_{2} \cdot i_{h}} \mid A_{i_{1} i_{2} \cdots i_{h}}\right)\right\}$

$$
\left\{X_{i_{1} i_{2} \cdots i_{h}}\right\} \in E_{\lambda}\left(k_{i_{1} i_{2} \cdots i_{h}} ; D^{(h)}(A)\right),
$$

for $h=1,2, \cdots, m-1$. Moreover the stochastic variable $X$ is distributed in $g(x ; k \mid \lambda)$.

We shall give here a few examples which are important in our practical applications.

Example 3.1. Binomial distribution. This is the case when each stochastic variable $X_{i_{1} i_{2} \cdots i_{m}}$ is distributed according to the distribution

$$
\begin{aligned}
\operatorname{Pr} .\left\{X_{i_{1} i_{2} \cdots i_{h}}=x\right\} & =\left(\begin{array}{c}
k_{i_{1} i_{2} \cdots i_{m}} \\
x
\end{array}\right) p^{x}(1-p)^{k_{i_{1} \cdots i_{m}-x}} \\
& =g_{B}\left(x ; k_{i_{1} i_{2} \cdots i_{m}} \mid A_{i_{1} i_{2} \cdots i_{m}}\right), \quad \text { say, }
\end{aligned}
$$

for $x=0,1, \cdots, k_{i_{1} i_{2} \cdots i_{n} n}$. The transformation of the parameter $p$ into $\lambda$ by means of $\log \{p /(1-p)\}=\lambda$ reduces (3.4) to the exponential type (3.1) with

$$
\begin{gathered}
b(\lambda)=\log \left(e^{\lambda}+1\right)^{-1}, \\
a_{k_{i 1 i 2 \cdots i m}}(x)=\left(\begin{array}{c}
k_{i_{1} i_{2} \cdots i m} \\
x
\end{array}\right)
\end{gathered}
$$

and $d \mu_{i_{1} i 2 \cdots i_{m}}(x)=1$, for $x=0,1,2, \cdots, k_{i_{1} i_{2} \cdots i_{m}}$ and is equal to zero otherwise.

Example 3.2. Negative binomial distribution. This is the case when each $X_{i_{1} i_{2} \cdots i_{m}}$ is distributed according to the distribution 


$$
\begin{aligned}
\operatorname{Pr} .\left\{X_{i_{1} i_{2} \cdots i_{m}}=x\right\} & =\left(\begin{array}{c}
k_{i_{1} i_{2} \cdots i_{m}}+x-1 \\
x
\end{array}\right) p^{x}(1-p)^{k_{2_{12} 2_{2} \cdots i_{m}}} \\
& =g_{v_{B}}\left(x ; k_{i_{1} i_{2} \cdots i_{m}} \mid A_{i_{1} i_{2} \cdots i_{m}}\right)
\end{aligned}
$$

for $x=0,1,2, \cdots$. The transformation of the parameter $p$ into $\lambda$ by means of $\lambda=\log p$ reduces (3.7) to the exponential type (3.1) with

$$
\begin{aligned}
b(\lambda) & =\log \left(1-e^{\lambda}\right), \\
a_{k_{i_{1} i_{2} \cdots i_{m}}}(x) & =\left(\begin{array}{c}
k_{i_{1} i_{2} \cdots i_{m}}+x-1 \\
x
\end{array}\right)
\end{aligned}
$$

and $d \mu_{k_{11 i 2} \cdots i m}(x)=1$, for $x=0,1,2, \cdots$, and is equal to zero otherwise.

Example 3.3. Poission distribution. This is the case when each $X_{i_{1} i_{2} \cdots i_{m}}$ is distributed according to the distribution

$$
\begin{aligned}
\operatorname{Pr} .\left\{X_{i_{1} i_{2} \cdots i_{m}}=x\right\} & =\exp \left\{-k_{i_{1} i_{2} \cdots i_{m}} \theta\right\}\left(k_{i_{1} i_{2} \cdots i_{m}} \theta\right)^{x} / x ! \\
& =g_{p}\left(x ; k_{i_{1} i_{2} \cdots i_{m}} \mid A_{i_{1} i_{2} \cdots i_{m}}\right)
\end{aligned}
$$

for $x=0,1,2, \cdots$. The transformation of the parameter $\theta$ into $\lambda$ by means of $\log \theta=\lambda$ reduces (3.10) to the exponential type distribution with

$$
\begin{gathered}
b(\lambda)=-e^{\lambda}, \\
a_{k_{i_{1} i 2 \cdots i m}}(x)=\frac{k_{i_{1 i 2} \cdots i_{m}}^{x}}{x !} .
\end{gathered}
$$

Remarks on possible modifications of notations on stochastic variables $\left\{X_{i_{1} i_{2} \cdots i_{h}}\right\}$ and $\left\{k_{i_{1} i_{2} \cdots i_{h}}\right\}$ should be added here, similarly as we have made on the last part of section 2. Indeed we may use, in some cases when we consider it adequate and useful, the notations $X_{i_{1} i_{2} \cdots i_{h} \ldots}, k_{i_{1} i_{2} \cdots i_{h} \ldots}, x_{\ldots \ldots .}$ and $k \ldots . .$. instead of the original notations $X_{i_{1} i_{2} \cdots i_{h}}, k_{i_{1} i_{2} \cdots i_{h}}, x$ and $k$ just introduced respectively.

We proceed to introduce

Definition 3. A set of $q_{1} q_{2} \cdots q_{k}$ stochastic variables $\left\{X_{i_{1} i_{2} \cdots i_{m}}\right\}\left(i_{h}=1,2, \cdots, q_{h}\right.$; $h=1,2, \cdots, m)$ is said to have an m-stage distributional pattern of one parameter generalised exponential type distributions with the set of additive parameters $\left\{k_{i_{1} i_{2} \cdots i_{\nu}}^{(\nu)}\right\}$ $(\nu=1,2, \cdots, m)$ when the following conditions are satisfied:

(1) The set of $q_{1}$ stochastic variables $\left\{X_{i_{1}}\right\}\left(i_{1}=1,2, \cdots, q_{1}\right)$ has a joint probability element

$$
\operatorname{Pr} \cdot\left\{\prod_{i_{1}=1}^{q_{1}} X_{i_{1}}=x_{i_{1}}\right\}=\prod_{i_{1}=1}^{q_{1}} g^{(1)}\left(x_{i_{1}} ; k_{i_{1}}^{(1)} \mid A_{i_{1}}\right) d \mu^{(1)}\left(x_{i_{1}}\right) .
$$

(2) The conditional joint probability element of $q_{h}$ stochastic variables $\left\{X_{i_{1} i_{2} \cdots i_{h-1} i_{h}}\right\}$ $\left(i_{h}=1,2, \cdots, q_{h}\right)$ under the condition that the stochastic variable $X_{i_{1} i_{2} \cdots i_{h-1}}$ is assigned as to be equal to $x_{i_{1} i_{2} \cdots i_{h-1}}$ is given by

$$
\begin{aligned}
\operatorname{Pr} . & \left\{\prod_{i_{h}=1}^{q h}\left(X_{i_{1} i_{2} \cdots i_{h}}=x_{i_{1} i_{2} \cdots i_{h}}\right) \mid X_{i_{1} i_{2} \cdots i_{h-1}}=x_{i_{1} i_{2} \cdots i_{h-1}}\right\} \\
& =\frac{\prod_{i_{h}=1}^{q h} g_{i_{12} \cdots i_{h-1}}^{(h)}\left(x_{i_{1} i_{2} \cdots i_{h}} ; k_{i_{1} i_{2} \cdots i_{h}}^{(h)} \mid A_{i_{1} i_{2} \cdots i_{h}}\right) d \mu_{i_{1} \cdots i_{h-1}}^{(h)}\left(x_{i_{1} i_{2} \cdots i_{h}}\right)}{g_{i_{1} i_{2} \cdots i_{h-1}}^{(h)}\left(x_{i_{1} i_{2} \cdots i_{h-1}} ; k_{i_{1} i_{2} \cdots i_{h-1}}^{(h)} \mid A_{i_{1} i_{2} \cdots i_{h}-1}\right) d \mu_{i_{1} \cdots i_{h-1}}^{(h)}\left(x_{i_{1} i_{2} \cdots i_{h-1}}\right)} \\
& =d g_{i_{1} i_{2} \cdots i_{h-1}}^{(h)}\left(\left(x_{i_{1} i_{2} \cdots i_{h}}\right)\left|x_{i_{1} i_{2} \cdots i_{h-1}} ; k_{i_{1} i_{2} \cdots i_{h}}^{(h)}\right| A_{i_{1} i_{2} \cdots i_{h}}\right), \quad \text { say. }
\end{aligned}
$$


(3) The conditional joint probability element of $q_{h_{1}} q_{h_{1}+1} \cdots q_{h_{2}}$ stochastic variables $\left\{X_{i_{1} i_{2} \cdots i_{h}}\right\} \quad\left(i_{j}=1,2, \cdots, q_{j} ; j=h_{1}, h_{1}+1, \cdots, h_{2}\right)$ under the condition that $X_{i_{1} i_{2} \cdots i_{h_{1}-1}}$ $=x_{i_{1} i_{2} \cdots h_{i-1}}$, for $i_{j}=1,2, \cdots, q_{j}, j=1,2, \cdots, h_{1}-1$, is given by

$$
\begin{aligned}
\operatorname{Pr} . & \left\{E^{(2)}\left(h_{1}, h_{2}\right) \mid E^{(1)}\left(h_{1}-1\right)\right\} \\
& =\prod_{i_{h_{1}}=1}^{q h_{1} 1} \prod_{h_{1}+1=1}^{q_{h_{1}+1}+1} \cdots \prod_{i_{2}=1}^{q_{h_{2}}} d G\left(i_{h_{1}} i_{h_{1}+1} \cdots i_{h_{2}} \mid i_{1} i_{2} \cdots i_{h_{1}-1}\right),
\end{aligned}
$$

where the left-hand side of (3.15) denotes the probability element of the occurance of the event $E^{(2)}\left(h_{1}, h_{2}\right)$ under the condition that the event $E^{(1)}\left(h_{1}-1\right)$ occurs where

$$
\begin{aligned}
& E^{(2)}\left(h_{1}, h_{2}\right): \prod_{i_{h_{1}=1}}^{q_{h_{1}}} \cdots \prod_{i_{h_{2}=1}}^{q_{h_{2}}}\left(X_{i_{1} \cdots i_{h_{1}-1} i_{h_{1} \cdots h_{2}}}=x_{i_{1} \cdots i_{h_{1}-1} i_{h_{1} \cdots h_{2}}}\right), \\
& E^{(1)}\left(h_{1}-1\right): \prod_{i_{1}=1}^{q_{1}} \cdots \prod_{i_{h_{1}-1}=1}^{q_{h_{1}-1}}\left(X_{i_{1} i_{2} \cdots i_{h_{1}-1}}=x_{i_{1} i_{2} \cdots i_{h_{1}-1}}\right)
\end{aligned}
$$

and in the right-hand side of (3.15) we have put

$$
\begin{aligned}
& d G\left(i_{h_{1}} i_{h_{1}+1} \cdots i_{h_{2}} \mid i_{1} i_{2} \cdots i_{h_{1}-1}\right) \\
& \quad=\prod_{j=h_{1}}^{h_{2}} d g_{i_{12} \cdots i_{j-1}}^{(j)}\left(\left(x_{i_{1} \cdots i_{h_{1}-1 i_{1} \cdots i_{j}}}\right)\left|x_{i_{1} \cdots i_{j-1}} ; k_{i_{1 i 2} \cdots i_{j}}^{(j)}\right| A_{i_{1} i_{2} \cdots i_{j}}\right) .
\end{aligned}
$$

It is readily seen that the stochastic scheme defined by Definition 2 is an extremely specialised case of the general scheme introduced in Definition 3. Between these two cases there are a lot of types of multistage distributional patterns which are worth while to be noticed. For a systematic description of these situations it is adequate to introduce two fundamental notions, homegeneity and inheritance, in the following way :

Definition 4. In a set of $q_{1} q_{2} \cdots q_{k}$ stochastic variables $\left\{X_{i_{1} i_{2} \cdots i_{m}}\right\}\left(i_{h}=1,2, \cdots, q_{h}\right.$; $h=1,2, \cdots, m)$ having a multistage distributional pattern of generalised exponential type distribution with the set of additive parameter $\left\{k_{i_{1} i_{2} \cdots i_{\nu}}^{(\nu)}\right\}(\nu=1,2, \cdots, m)$, a set of $q_{h+1} \cdots q_{h+d}$ stochastic variables $\left\{X_{i_{1} i_{2} \cdots i_{h} \cdots i_{h+d}}\right\}\left(i_{j}=1,2, \cdots, q_{j} ; j=h+1, \cdots, h+d\right)$ is said to be homogeneous in the d-depth in the set $A_{i_{1} i_{2} \cdots i_{h}}$ when there exists a sequence of functions $\left\{g_{i_{12} \cdots i_{h}}^{(j)}\right\}$ and $\left\{d \mu_{i 1 i_{2} \cdots i_{h}}^{(j)}\right\}$ such that

$$
\begin{aligned}
& d g_{i_{1} i_{2} \cdots i_{j-1}}^{(j)}\left(\left(x_{i_{1} i_{2} \cdots i_{j}}\right)\left|x_{i_{1} i_{2} \cdots i_{j-1}} ; k_{i_{1} i_{2} \cdots i_{j}}^{(j)}\right| A_{i_{1} i_{2} \cdots i_{j}}\right) \\
& \quad=d g_{i_{1 i 2}^{(j) \cdots i_{h}}}\left(\left(x_{i_{1} i_{2} \cdots i_{j}}\right)\left|x_{i_{1} i_{2} \cdots i_{j-1}} ; k_{i_{1} i_{2} \cdots i_{j}}^{(j)}\right| A_{i_{1} i_{2} \cdots i_{j}}\right),
\end{aligned}
$$

for $i_{j}=1,2, \cdots, q_{j} ; j=h+1, \cdots, h+d$.

Definition 5. A set of $q_{h+1} \cdots q_{h+d}$ stochastic variables $\left\{X_{i_{1} i_{2} \cdots i_{h-1} i_{h} \cdots i_{h+d}}\right\}\left(i_{j}=1,2\right.$, $\left.\cdots, q_{j} ; j=h+1, \cdots, h+d\right)$ is said to be inherited with the d-depth in the set $A_{i_{1} i_{2} \cdots i_{h}}$ when the following two conditions are satisfied:

$\left(1^{\circ}\right) \quad k_{i_{1 i 2} \cdots i_{j-1}}^{(j)}=k_{i_{1 i 2} \cdots i_{j-1}}^{(j-1)}, \quad(j=h+1, \cdots, h+d)$

$\left(2^{\circ}\right) \quad g_{i_{1 i 2} \cdots i_{j-1}}^{\langle j\rangle}\left(x_{i_{1} i_{2} \cdots i_{j-1}} ; k_{i_{1} i_{2} \cdots i_{j-1}}^{(j)} \mid A_{i_{1} i_{2} \cdots i_{j-1}}\right) d \mu_{i_{112} \cdots i_{j-1}}^{\langle j\rangle}\left(x_{i_{1} i_{2} \cdots i_{j-1}}\right)$

$$
=g_{i_{112} \cdots i_{j-2}}^{(j-1)}\left(x_{i_{1} i_{2} \cdots i_{j-1}} ; k_{i_{1} i_{2} \cdots i_{j-1}}^{(j-1)} \mid A_{i_{1} i_{2} \cdots i_{j-1}}\right) d \mu_{i_{1} i_{2} \cdots i_{j-2}}^{(j-1)}\left(x_{i_{1} i_{2} \cdots i_{j-1}}\right)
$$

for every $x_{i_{1} i_{2} \cdots i_{j-1}}$ and $k_{i 12 q^{(j)} i_{j-1}}$ in their domains of definition, for $j=h+1, \cdots, h+d$.

In virtue of Definitions 3,4 and 5 it is immediate to see

Proposition 3. The set of $q_{1} q_{2} \cdots q_{m}$ stochastic variables $\left\{X_{i_{1} i_{2} \cdots i_{m}}\right\} \in E_{\lambda}\left(k_{i_{1} i_{2} \cdots i_{m}}\right.$; $\left.D^{(m)}(A)\right)$ is both homogeneous and inherited in the m-depth in the set $A$. 
Proof. This can be seen by an interpretation to the effect that the assumption (3.2) implies

$$
\begin{gathered}
k_{i_{12} \cdots i_{h}}^{(h)}=k_{i_{1} i_{2} \cdots i_{h}}, \\
g_{i_{1} i_{2} \cdots i_{h-1}}^{(h)}\left(x_{i_{1} i_{2} \cdots i_{h}} ; k_{i_{1} i_{2} \cdots i_{h}}^{(h)} \mid A_{i_{1} i_{2} \cdots i_{h}}\right) d \mu_{i_{1} \cdots i_{h-1}}^{(h)}\left(x_{i_{1} i_{2} \cdots i_{h-1}}\right) \\
=g\left(x_{i_{1} i_{2} \cdots i_{h}} ; k_{i_{1} i_{2} \cdots i_{h}} \mid A_{i_{1} i_{2} \cdots i_{h}}\right) d \mu\left(x_{i_{1} i_{2} \cdots i_{h-1}}\right)
\end{gathered}
$$

for $i_{j}=1,2, \cdots, q_{j} ; j=1,2, \cdots, h ; h=1,2, \cdots, m$.

Particular examples of two and three stage distributional patterns are explained by adopting various cominations of binomial, negative binomial and Poisson distributions in defining functions in Definition 3.

Example 3.4. Two stage distributional patterns. Nine sets of two stage distributional patterns can be obtained from all the possible permutations by adopting one of the three fundamental probability density functions as shown in the following Table. We may and we shall denote these two stage distributional patterns by $B \times B$, $B \times N B, B \times P, N B \times B, N B \times N B, N B \times P, P \times B, P \times N B$ and $P \times P$.

Table 1. Probability Element Function (PEF) in Two stage Distributional Pattern.

$\begin{array}{cc}\frac{\mathrm{PEF}}{\text { Illustrative Examples }} \\ d g^{(1)}\left(x ; k_{i_{1}}^{(1)} \mid A_{i_{1}}\right) & B, N B, P \\ d g_{i_{1}}^{(2)}\left(\left(x_{i_{1} i_{2}}\right)\left|x_{i_{1}} ; k_{i_{1} i_{2}}^{(2)}\right| A_{i_{1} i_{2}}\right) & B, N B, P\end{array}$

A direct consequence of Definitions 3 and 4 yields us the following observations:

(i) Homogeneity. Two stage distributional pattern is homogeneous if and only if there is a function such that

$$
d g_{i_{1}}^{(2)}\left(\left(x_{i_{1} i_{2}}\right)\left|x_{i_{1}} ; k_{i_{112}}^{(2)}\right| A_{i_{1} i_{2}}\right)=d g^{(2)}\left(\left(x_{i_{1} i_{2}}\right)\left|x_{i_{1}} ; k_{i_{112}}^{(2)}\right| A_{i_{1} i_{2}}\right)
$$

for $i_{j}=1,2, \cdots, q_{j} ; j=1,2$.

(ii) Inheritance. Two stage distributional pattern is inherited if and only if the following two conditions are satisfied:

$\left(1^{\circ}\right) \quad k_{i_{1}}^{(2)}=k_{i_{1}}^{(1)} \quad\left(i_{1}=1,2, \cdots, q_{1}\right)$

$\left(2^{\circ}\right) \quad g_{i_{1}}^{(2)}\left(x_{i_{1}} ; k_{i_{12}}^{(2)} \mid A_{i_{1} i_{2}}\right)=g^{(1)}\left(x_{i_{1}} ; k_{i_{1} i_{2}}^{(2)} \mid A_{i_{1} i_{2}}\right)$ for $i_{1}=1,2, \cdots, q_{j} ; j=1,2$.

Example 3.5. Three stage distributional patterns. Twenty seven sets of three stage distributions such as $B \times B \times B, B \times B \times N B, N B \times P \times B$ and so on can be obtained from all the possible permutations of the fundamental probability density functions as shown in the following Table 2 .

Table 2. Probability Element Function (PEF) in Three stage Distributional Pattern.

\begin{tabular}{ll} 
PEF & Illustrative Examples \\
\hline$d g^{(1)}\left(x ; k_{i_{1}}^{(1)} \mid A_{i_{1}}\right)$ & $B, N B, P$ \\
$d g_{11}^{(2)}\left(\left(x_{i_{1} i_{2}}\right)\left|x_{i_{1}} ; k_{i_{12}}^{(2)}\right| A_{i_{1} i_{2}}\right)$ & $B, N, P$ \\
$d g_{i_{12}(3)}^{(3)}\left(\left(x_{i_{1} i_{2} i_{3}}\right)\left|x_{i_{1} i_{2}} ; k_{i_{1} i_{2} i_{3}}^{(3)}\right| A_{i_{1} i_{2} i_{3}}\right)$ & $B, N B, P$ \\
\hline
\end{tabular}


In conclusion of section 3 let us introduce some notations for the conditional expected values of stochastic variables. Let $z=\left\{z_{i_{1} i_{2} \cdots i_{h}}\right\}\left(i_{j}=1,2, \cdots q_{j} ; j=1,2, \cdots, h\right)$ be a set of stochastic variables and let $P(z)$ be the multidimensional probability distribution function of $z$. Let $H(z)$ be a function of $z$ with its domain of definition $\mathfrak{D}(H)$. For an assigned set of non-negative integers $\left(i_{1}, i_{2}, \cdots, i_{h}\right)$, an assigned value $y$ and an assigned function $u$, let us denote by $\mathfrak{D}_{u\left(i_{1} i_{2} \cdots i_{h}\right)(y)}(H)$ the subset of $\mathfrak{D}(H)$ where $u\left(z_{i_{1} i_{2} \cdots i_{h}}\right)$ has the value $y$.

Under these preparations, let us introduce a set of the conditional expectation of the stochastic variable $u(z)$ such that

$$
E^{*}\{H\}=\frac{\int_{z \in \mathscr{D}(H)} H(z) d P(z)}{\int_{z \in \mathscr{D}(H)} d P(z)}
$$

and

$$
E_{u\left(i_{1} i_{2} \cdots i_{h}\right)(y)}^{*}\{H\}=\frac{\int_{z \in \mathbb{L}_{u\left(i_{1} \cdots i_{h}\right)(y)(H)}} H(z) d P(z)}{\int_{z \in \mathscr{D}_{u\left(i_{1} \cdots i_{h}\right)(y)}(H)} d P(z)},
$$

provided that the right-hand side of (3.23) and that of (3.24) exist respectively.

We use also the notion $E_{G}^{*}\{H\}$ to specify an underlying probability function $G$ such as binomial $(B)$, negative binomial $(N B)$ and Poisson $(P)$ distributions. For instance $E_{B}^{*}\{H\}, E_{N B}^{*}\{H\}$ and $E_{P}^{*}\{H\}$ will be used to express the conditional expectation of $H$.

\section{$\S 4$. Reproductive function in additive family of one parameter exponential type distributions.}

Let us introduce

Definition 6. A function $\phi(x)$ is said to be reproductive in an additive family of one parameter exponential type distributions $\{g(x ; k)\}$ with additive parameter $k$, if there exists a set of a function $\phi(x)$, a non-vanishing function $\varphi(k)$, a transformation $T$ of $x$, a transformation $S$ of $k$ and a non-vanishing function $c(\phi)$, which is independent of $k$ and $x$, such that, for any assigned set of $x$ and $k$ belonging to their respective domain of definition for specifying $g(x ; k)$, we have

$$
\phi(x) g(x ; k) d \mu(x)=\varphi(k) c(\phi) g(T x ; S k) d \mu(T x)
$$

with the following properties:

$\left(1^{\circ}\right)$ For any set of $x_{1}, x_{2}, \cdots, x_{q-1}$ and $x_{q}$ belonging to the domain of definition $g(x ; k)$ and for any positive integer $i$ in $1 \leqq i \leqq q$, we have

$$
T\left(\sum_{j=1}^{q} x_{j}\right)=\sum_{j=1}^{i-1} x_{j}+T x_{i}+\sum_{l=i+1}^{q} x_{l} .
$$

$\left(2^{\circ}\right)$ For any set of non-negative integers $k_{1}, k_{2}, \cdots, k_{q-1}$ and $k_{q}$ and any positive integer $i$ in $1 \leqq i \leqq q$, we have 


$$
S\left(\sum_{j=1}^{q} k_{j}\right)=\sum_{j=1}^{i-1} k_{j}+S k_{i}+\sum_{l=i=1}^{q} k_{l} .
$$

We observe

Corollary 1. The function $\phi^{-j}(x)=x(x-1) \cdots(x-j+1)$ is reproductive in additive family of one parameter exponential type distributions $\{g(x ; k)\}$ with additive parameter $k$ when $g(x ; k)$ is one of the three fundamental distributions, namely, binomial, negative binomial and Poisson ones. Indeed we have

(a) Binomial distribution $g_{B}(x ; k)$. We have

$$
\phi^{-j \geq}(x) g_{B}(x ; k)=\varphi_{B}(k) c_{B}(\phi) g_{B}\left(T_{B} x ; S_{B} k\right),
$$

where we have put

(b) Negative binomial distribution $g_{N B}(x ; k)$. We have

$$
\phi^{[j]}(x) g_{N B}(x ; k)=\varphi_{N B}(k) c_{N B}(\phi) g_{N B}\left(T_{N B} x ; S_{N B} k\right),
$$

where we have put

$$
\begin{gathered}
\varphi_{N B}(k)=k(k+1) \cdots(k+j-1), \\
c_{N B}(\phi)=p^{j}(1-p)^{-j}, \\
T_{N B} x=x-j, \quad S_{N B}=k+j .
\end{gathered}
$$

(c) Poisson distribution $g_{p}(x ; k)$. We have

$$
\phi^{[j\urcorner}(x) g_{P}(x ; k)=\varphi_{P}(k) c_{P}(\phi) g_{P}\left(T_{P} x ; S_{P} k\right),
$$

where we have put

$$
\begin{gathered}
\varphi_{P}(k)=k^{j}, \\
c_{P}(\phi)=\lambda^{j}, \\
T_{P} x=x-j, \quad S_{P} k=k .
\end{gathered}
$$

It is convenient to have

Definition 7. A function $\phi(x)$ is said to be reproductive in a multistage distributional pattern of one parameter generalised exponential type distributions with the set of additive parameters $\left\{k_{i_{1} i_{2} \cdots i_{2}}^{(\nu)}\right\}(\nu=1,2, \cdots, m)$, or simply reproductive in the system $D^{(m)}(A)$, when all the concurrence-probability distribution density functions are reproductive, that is to say, the relations hold true

$$
\begin{aligned}
& \phi(x) g_{i_{1} i_{2} \cdots i_{h-1}}^{(h)}\left(x_{i_{1} i_{2} \cdots i_{h}} ; k_{i_{112} \cdots i_{h}}^{(h)} \mid A_{i_{1} i_{2} \cdots i_{h}}\right) d \mu_{i_{1} \cdots i_{h-1}}^{(h)}\left(x_{i_{1} i_{2} \cdots i_{h}}\right) \\
& =\varphi_{i_{1} i_{2} \cdots i_{h-1}}^{(h)}\left(k_{i_{1} i_{2} \cdots i_{h}}^{(h)}\right) c_{i_{12} i_{2} \cdots i_{h-1}}^{(h)}(\phi) g_{i_{112} \cdots i_{h-1}}^{(h)}\left(T_{i_{1} i_{2} \cdots i_{h-1}}^{(h)} x_{i_{1} i_{2} \cdots i_{h}} ;\right. \\
& \left.S_{i_{1} i_{2} \cdots i_{h-1}}^{(h)} k_{i_{1} i_{2} \cdots i_{h}}^{(h)}\right) d \mu_{i_{1} i_{2} \cdots i_{h-1}}^{(h)}\left(T_{i_{1} i_{2} \cdots i_{h-1}}^{(h)} x_{i_{1} i_{2} \cdots i_{h}}\right),
\end{aligned}
$$

where the functions $c_{i_{1 i 2} \cdots i_{h-1}}^{(h)}(\phi)$, transformations $T_{i_{1 i 2} \cdots i_{n-1}}^{(h)}$ and $S_{i_{112} \cdots i_{h-1}}^{(h)}$ satisfy the conditions similar to those enunciated in Definition 6 . 


\section{$\S 5$. The conditional expectation of $\phi$-dispersion for reproductive $\phi$-function.}

It is the purpose of this section to evaluate various conditional expectations of $\phi$-dispersions such as $E^{*}\left\{\phi_{d}[A]\right\}$ and $E_{c}^{*}\left\{\dot{\phi}_{d}\left[A_{i_{1} i_{2} \cdots i_{h}}\right]\right\}$ for $d \geqq 1$ and for a certain set of conditions $c$ such as specifying underlying probability density function. In order to make clear the essential aspects of our evaluations which are valid for a fairly general situation, let us be concerned with the simplest two examples.

Proposition 4. For a reproductive function $\phi$ in the decomposition system $D^{(1)}(A)$ we have

$$
E^{*}\left\{\phi_{1}[A]\right\}=\frac{\sum_{i=1}^{q} \varphi\left(k_{i}\right)}{\varphi\left(\sum_{i=1}^{q} k_{i}\right)}=\frac{\sum_{i=1}^{q} \varphi\left(k_{i}\right)}{\varphi(k)} .
$$

Proof. We have, in virtue of definition,

$$
E^{*}\left\{\phi_{1}[A]\right\}=\int_{\left\{x_{i}\right\} \equiv \mathcal{D} \phi_{1}[A]} \ldots \iint_{i=1}^{\sum_{i=1}^{q} \phi\left(x_{i}\right)} \frac{q}{\phi(x)} \prod_{i=1}^{q} g\left(x_{i} ; k_{i}\right) d \mu\left(x_{i}\right),
$$

where the domain of integration $\mathscr{D} \phi_{1}[A]$ is the set of all non-negative integers for which $\phi_{1}[A]$ is defined and the symbol $\left\{x_{i}\right\} \in \mathscr{D} \phi_{1}[A]$ denotes the set of $\left\{x_{i}\right\}(i=1,2$, $\cdots, q)$ such that $\left(x_{1}, x_{2}, \cdots, x_{q}\right)$ belongs to $\mathscr{D} \phi_{1}[A]$.

Since the function $\phi$ is reproductive in $D^{(1)}(A)$, we have, for $i=1,2, \cdots, q$,

$$
\begin{aligned}
\phi\left(x_{i}\right) \prod_{h=1}^{q} g\left(x_{h} ; k_{h}\right) d \mu\left(x_{h}\right) & =\phi\left(x_{i}\right) g\left(x_{i} ; k_{i}\right) d \mu\left(x_{i}\right) \prod_{h \neq i} g\left(x_{h} ; k_{h}\right) d \mu\left(x_{h}\right) \\
& =\varphi\left(k_{i}\right) c(\phi) g\left(T x_{i} ; S k_{i}\right) d \mu\left(T x_{i}\right) \prod_{h \neq i} g\left(x_{h} ; k_{h}\right) d \mu\left(x_{h}\right),
\end{aligned}
$$

in virtue of (4.1). In view of (4.2) and (4.3), it ts readily observed that, when $\left(x_{1}, x_{2}\right.$, $\left.\cdots, x_{q}\right)$ runs through the set for which $\sum_{i=1}^{q} x_{i}$ is equal to an assigned value $x^{*}$, the point $\left(x_{1}, x_{2}, \cdots, x_{i-1}, T x_{i}, x_{i+1}, \cdots, x_{q}\right)$ belongs to the set for which its sum is equal to $T\left(\sum_{i=1}^{q} x_{i}\right)=T x^{*}$ for any non-negative integer $i$ in $1 \leqq i \leqq q$, and also that the converse is true. After summing up the probability elements (5.3) with respect to $d \mu\left(x_{1}\right)$ $\cdots d \mu\left(x_{q}\right)$ over the set of $\left(x_{1}, x_{2}, \cdots, x_{q}\right)$ for which $x \equiv \sum_{i=1}^{q} x_{i}$ is assigned as a particular value, we have

$$
\varphi\left(k_{i}\right) c(\phi) g\left(T\left(\sum_{i=1}^{q} x_{i}\right) ; S\left(\sum_{i=1}^{q} k_{i}\right)\right) d \mu\left(T\left(\sum_{i=1}^{q} x_{i}\right)\right),
$$

which, apart from $\varphi\left(k_{i}\right)$, is independent of $i$. Now again the fact that $\phi(x)$ is reproductive implies, since $c(\phi) \varphi(k) \neq 0$,

$$
g(T x ; S x) d \mu(T x)=\frac{\phi(x) g(x ; k) d \mu(x)}{c(\phi) \varphi(k)},
$$

which, in combination with (5.4), leads us to

$$
E^{*}\left\{\phi_{1}[A]\right\}=\frac{\sum_{i=1}^{q} \varphi\left(k_{i}\right)}{\varphi(k)} \int_{x=\left\{\phi_{1}[A]\right.} g(x ; k) d \mu(x)
$$


as we were to prove.

$$
\times-\int_{x \in \mathscr{D} \phi_{1}[A]} g(x ; k) d \mu(x)=\frac{\sum_{i=1}^{q} \varphi\left(k_{i}\right)}{\varphi(k)},
$$

Example 5.1. (a) Binomial distribution

$$
E_{B}^{*}\left\{\phi_{1}^{[j-}[A]\right\}=\frac{\sum_{i=1}^{q} \varphi_{B}\left(k_{i}\right)}{\varphi_{B}(k)}=\frac{\sum_{i=1}^{q} k_{i}\left(k_{i}-1\right) \cdots\left(k_{i}-j+1\right)}{k(k-1) \cdots(k-j+1)} .
$$

(b) Negative binomial distribution

$$
E_{N B}^{*}\left\{\phi_{1}^{[j]}[A]\right\}=\frac{\sum_{i=1}^{q} \varphi_{N B}(k)}{\varphi_{N B}(k)}=\frac{\sum_{i=1}^{q} k_{i}\left(k_{i}+1\right) \cdots\left(k_{i}+j-1\right)}{k(k+1) \cdots(k+j-1)} .
$$

(c) Poisson distribution

$$
E_{P}^{*}\left\{\phi_{1}^{[j]}[A]\right\}=\frac{\sum_{i=1}^{q} \varphi_{P}\left(k_{i}\right)}{\varphi_{P}(k)}=\frac{\sum_{i=1}^{q} k_{i}^{j}}{k^{j}} .
$$

Proposition 5. For a reproductive function $\phi$, in the decomposition system $D^{(2)}(A)$, we have

$$
E *\left\{\phi_{2}[A]\right\}=\sum_{i_{1}=1}^{q_{1}} \frac{\varphi^{(1)}\left(k_{i 1}^{(1)}\right)}{\varphi^{(1)}\left(k^{(1)}\right)} \sum_{i_{2}=1}^{q_{2}} \frac{\varphi_{i_{1}}^{(1)}\left(k_{i i_{2}}^{(2)}\right)}{\varphi_{i_{1}}^{(2)}\left(k_{i 1}^{(2)}\right)} .
$$

Proof. The proof is quite similar to that of Proposition 3. First we note that, for each pair $\left(i_{1}, i_{2}\right)$, we have

$$
\begin{aligned}
& \phi\left(x_{i_{1} i_{2}}\right) \prod_{j=1}^{q_{2}} g_{i_{1}}^{(2)}\left(x_{i_{1} j} ; k_{i_{1} j}^{(2)}\right) d \mu_{i_{1}}^{(2)}\left(x_{i_{1} j}^{(2)}\right) \\
& \quad=\phi\left(x_{i_{1} i_{2}}\right) g_{i_{1}}^{(2)}\left(x_{i_{1} i_{2}} ; k_{i_{1} i_{2}}^{(2)}\right) d \mu_{i_{1}}^{(2)}\left(x_{i_{1 i 2}}^{(2)}\right) \prod_{j \neq i_{2}} g_{i_{1}}^{(2)}\left(x_{i_{1 j} j} ; k_{i_{1} j}^{(2)}\right) d \mu_{i_{1}}^{(2)}\left(x_{i_{1} j}^{(2)}\right) \\
& \quad=c^{(2)}(\phi) \varphi_{i_{1}}^{(2)}\left(k_{i_{1} i_{2}}^{(2)}\right) g_{i_{1}}^{(2)}\left(T_{i_{1}}^{(2)} x_{i_{1} i_{2}} ; S_{i_{1}}^{(2)} k_{i_{1} i_{2}}\right) d \mu_{i}^{(2)}\left(T_{i_{1}}^{(2)} x_{i_{1} i_{2}}^{(2)}\right) \cdot \prod_{h \neq i_{2}} g_{i_{1}}^{(2)}\left(x_{i_{1} h} ; k_{i_{1} h}^{(2)}\right) d \mu_{i_{1}}^{(2)}\left(x_{i_{1} j}^{(2)}\right),
\end{aligned}
$$

which yields us

$$
\begin{gathered}
\sum_{i_{2}=1}^{q_{2}} \phi\left(x_{i_{1} i_{2}}\right)\left\{\prod_{i_{2}=1}^{q_{2}} g_{i_{1}}^{(2)}\left(x_{i_{1} i_{2}} ; k_{i_{1} i_{2}}^{(2)}\right) d \mu_{i_{1}}^{(2)}\left(x_{i_{1} i_{2}}\right)\right\} \\
=c^{(2)}(\phi) \sum_{i_{2}=1}^{q_{2}} \varphi_{i_{1}}^{(2)}\left(k_{i_{1} i_{2}}^{(2)}\right) g_{i_{1}}^{(2)}\left(T_{i_{1}}^{(2)} x_{i_{1} i_{2}} ; S_{i_{1}}^{(2)} k_{i_{1} i_{2}}^{(2)}\right) d \mu_{2}^{(2)}\left(T_{i_{1}}^{(2)} x_{i_{1 i 2}}^{(2)}\right) \\
\cdot \prod_{h \neq i_{2}}\left\{g_{i_{1}}^{(2)}\left(x_{i_{1} h} ; k_{i_{1} h}^{(2)}\right) d \mu_{i_{1}}^{(2)}\left(x_{i_{1} h}\right)\right\} .
\end{gathered}
$$

In view of (4.2) and (4.3), after summing up the probability elements in (5.12), we have

$$
g_{i_{1}}^{(2)}\left(T_{i_{1}}^{(2)} x_{i_{1} i_{2}} ; S_{i_{1}}^{(2)} k_{i_{1 i} 2}^{(2)}\right) d \mu_{i_{1}}^{(2)}\left(T_{i_{1}}^{(2)} x_{i_{1} i_{2}}^{(2)}\right) \cdot \prod_{h \neq i_{2}}\left\{g_{i}^{(2)}\left(x_{i_{1} h} ; k_{i_{1} h}^{(2)}\right) d \mu_{i_{1}}^{(2)}\left(x_{i_{1} i_{2}}\right)\right\}
$$

with respect to $d \mu_{i_{1}}^{(2)}\left(x_{i_{1}}\right) \cdots d \mu_{i_{1}}^{(2)}\left(x_{i_{1} q_{2}}\right)$, we obtain

$$
g_{i_{1}}^{(2)}\left(T_{i_{1}}^{(2)} x_{i_{1}} ; S_{i_{1}}^{(2)} k_{i_{1}}^{(2)}\right) d \mu_{i_{1}}^{(2)}\left(T_{i_{1}}^{(2)} x_{i_{1}}\right)
$$

when $\left(x_{i_{11}}, \cdots, x_{i_{1} q_{2}}\right)$ runs through the set for which $\sum_{i_{2}=1}^{q_{2}} x_{i_{1} i_{2}}$ is equal to an assiged 
value $x_{i_{1}}$ (5.14) leads us to the probability element

$$
c^{(2)}(\phi)\left\{\sum_{i_{2}=1}^{q_{2}} \varphi_{i_{1}}^{(2)}\left(k_{i_{12}}^{(2)}\right)\right\} g_{i_{1}}^{(2)}\left(T_{i_{1}}^{(2)} x_{i_{1}} ; S_{i_{1}}^{(2)} k_{i_{1}}^{(2)}\right) d \mu_{i_{1}}^{(2)}\left(T_{i_{1}}^{(2)} x_{i_{1}}\right),
$$

Again in virtue of the fact that $\phi$ is reproductive, (5.15) turns out to be

$$
\frac{\sum_{i_{1}=1}^{q_{2}} \varphi_{i 1}^{(2)}\left(k_{i 1 i_{2}}^{(2)}\right)}{\varphi_{i_{1}}^{(2)}\left(k_{i_{1}}^{(2)}\right)}-\phi\left(x_{i_{1}}\right) g_{i_{1}}^{(2)}\left(x_{i_{1}} ; k_{i_{1}}^{(2)}\right) d \mu_{i_{1}}^{(2)}\left(x_{i_{1}}\right) .
$$

In the consequence we have

$$
\begin{aligned}
& E *\left\{\phi_{2}[A]\right\} \\
& =\iint_{\left\{x_{11 i_{2}}\right\} \equiv \mathcal{D} \phi_{2}[A]} \frac{\sum_{i_{1}=1}^{q_{1}} \sum_{i_{2}=1}^{q_{2}} \phi\left(x_{i_{1} i_{2}}\right)}{\phi(x)} \cdot \frac{\prod_{i_{1}-1}^{q_{1}} \prod_{i_{2}=1}^{q_{2}} g_{i_{1}}^{(2)}\left(x_{i_{1} i_{2}} ; k_{i i_{2}}^{(2)}\right) d \mu_{i_{1}}^{(2)}\left(x_{i_{1} i_{2}}\right)}{\prod_{i_{1}=1}^{q_{1}} g_{i_{1}}^{(2)}\left(x_{i_{1}} ; k_{i_{1}}^{(2)}\right) d \mu_{i_{1}}^{(2)}\left(x_{i_{1}}\right)} \\
& \cdot \prod_{i_{1}=1}^{q_{1}} g^{(1)}\left(x_{i_{1}} ; k_{i_{1}}^{(1)}\right) d \mu^{(1)}\left(x_{i_{1}}\right) \times \frac{1}{\operatorname{Pr} \cdot\left\{\mathfrak{D} \phi_{2}[A]\right\}} \\
& =\iint_{\left\{x_{\left.i_{1} i_{2}\right\}=\Phi \phi_{1}[A]} \ldots \ldots \ldots . .\right.} \frac{1}{\phi(x)} \sum_{i_{1}=1}^{q_{1}} \frac{\phi\left(x_{i_{1}}\right)}{\varphi_{i_{1}}^{(2)}\left(k_{i_{1}}^{(2)}\right)} \prod_{i_{1}=1}^{q_{1}} g^{(1)}\left(x_{i_{1}} ; k_{i_{1}}^{(1)}\right) d \mu^{(1)}\left(x_{i_{1}}\right) \\
& \cdot \sum_{i_{2}=1}^{q_{2}} \varphi_{i_{1}}^{(2)}\left(k_{i_{112}}^{(2)}\right) / \operatorname{Pr} .\left\{\left\{x_{i_{1} i_{2}}\right\} \in \mathscr{D} \phi_{2}[A]\right\} .
\end{aligned}
$$

Here we can deal with a transformation

$$
\phi\left(x_{i_{1}}\right) \prod_{i_{1}=1}^{q_{1}} g^{(1)}\left(x_{i_{1}} ; k_{i_{1}}^{(1)}\right): c^{(1)}(\phi) \varphi^{(1)}\left(k_{i_{1}}^{(1)}\right) g^{(1)}\left(T^{(1)} x_{i_{1}} ; S^{(1)} k_{i_{1}}^{(1)}\right) \prod_{h \neq i_{1}} g^{(1)}\left(x_{h} ; k_{h}^{(1)}\right)
$$

as in the proof of Proposition 5.

Consequently, after summing up the probability elements in (5.18) with respect to $d \mu^{(1)}\left(x_{1}\right) d \mu^{(1)}\left(x_{2}\right) \cdots d \mu^{(1)}\left(x_{q_{1}}\right)$ under the condition that $\left(x_{1}, x_{2}, \cdots, x_{q_{1}}\right)$ runs through the set for which $\sum_{i_{1}=1}^{g_{1}} x_{i_{1}}$ is equal to an assigned value $x$, we obtain that the sum of

$$
c^{(1)}(\phi) \sum_{i_{1}=1}^{q_{1}} \varphi^{(1)}\left(k_{i_{1}}^{(1)}\right) g^{(1)}\left(T^{(1)} x_{i_{1}} ; S^{(1)} k_{i_{1}}^{(1)}\right) d \mu^{(1)}\left(T x_{i_{1}}\right) \cdot \prod_{h \neq i_{1}} g^{(1)}\left(x_{h} ; k_{h}^{(1)}\right) d \mu^{(1)}\left(x_{h}\right)
$$

is equal to

$$
\frac{\sum_{i_{1}=1}^{q_{1}} \varphi^{(1)}\left(k_{i_{1}}^{(1)}\right)}{\varphi^{(1)}\left(k^{(1)}\right)} \phi(x) g^{(1)}\left(x ; k^{(1)}\right) d \mu^{(1)}(x),
$$

which leads us to the result to be proved.

Now we are in the position to enunciate a general result on the conditional expectation of $\phi$-dispersion.

Proposition 6. For a reproductive function $\phi$ in the decomposition system $D^{(d)}\left(A_{i_{1} i_{2} \cdots i_{h}}\right)$ we have

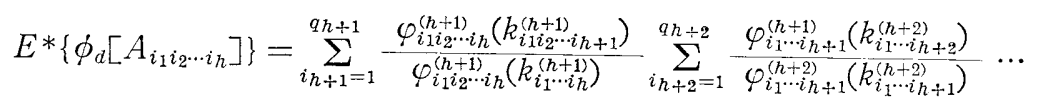




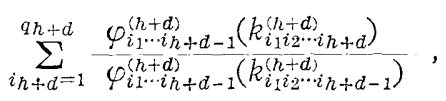

where the case $h=0$ can be interpretated as that

$$
\begin{aligned}
& E^{*}\left\{\phi_{d}[A]\right\}=\sum_{i_{1}=1}^{q_{1}} \frac{\varphi^{(1)}\left(k_{i 1}^{(1)}\right)}{\varphi^{(1)}\left(k^{(1)}\right)} \sum_{i_{2}=1}^{q_{2}} \frac{\varphi_{i 1}^{(2)}\left(k_{i 12}^{(2)}\right)}{\varphi_{i_{1}}^{(2)}\left(k_{i_{1}}^{(2)}\right)} \sum_{i_{3}=1}^{q_{3}} \frac{\varphi_{i 1 i_{2}}^{(3)}\left(k_{i_{12}}^{(3)}\right)}{\varphi_{i 1 i_{2}}^{(3)}\left(k_{i_{1}}^{(3)}\right)} \\
& \cdots \sum_{i_{d}=1}^{q_{d}} \frac{\varphi_{i 1 i_{2} \cdots i_{d-1}}^{(d)}\left(k_{i 1 i 2 \cdots i d}^{(d)}\right)}{\varphi_{i_{1 i} \cdots i d-1}^{(d)}\left(k_{i_{1} i_{2} \cdots i d-1}^{(d)}\right)} .
\end{aligned}
$$

Example 5.2. Two stage homogeneous distributional pattern. This is characterized by two systems of probability distribution elements $g^{(1)} d \mu^{(1)}$ in (3.13) and $g^{(2)} d \mu^{(2)}$ in (3.14), which, in this case, may and will be denoted by $G d \mu^{(1)} \times L d \mu^{(2)}$, where $G$ (global) and $L$ (local) correspond to $g^{(1)}$ and $g^{(2)}$ respectively.

In general we have for such an two stage homogeneous distributional pattern,

$$
E_{G \times L}^{*}\left\{\phi_{2}[A]\right\}=\sum_{i_{1}=1}^{q_{1}} \frac{\varphi_{G}\left(k_{i_{1}}^{(1)}\right)}{\varphi_{G}\left(k^{(1)}\right)} \sum_{i_{\mathbf{2}}=1}^{q_{2}} \frac{\varphi_{L}\left(k_{i_{1 i 2}}^{(2)}\right)}{\varphi_{L}\left(k_{i_{1}}^{(2)}\right)} .
$$

which turns out to the expression

$$
E_{G \times L}^{*}\left\{\phi_{2}[A]\right\}=\frac{1}{\varphi_{G}\left(k^{(2)}\right)} \sum_{i_{1}=1}^{q_{1}} \sum_{i_{2}=1}^{q_{2}} \varphi_{G}\left(k_{i_{112}}^{(2)}\right),
$$

particularly when two-stage distributional pattern is inherited, as can be readily seen from the fact that $\varphi_{G}(k)=\varphi_{L}(k)$ for every $k$ in their common domain of definition.

(a) Inherited binomial distributional pattern $(B \times B)$ (c. f. (4.5))

$$
E_{B \times B}^{*}\left\{\phi_{2}^{[j]}[A]\right\}=\sum_{i_{1}=1}^{q_{1}} \frac{\varphi_{B}\left(k_{1}^{(1)}\right)}{\varphi_{B}\left(k^{(1)}\right)} \sum_{i_{2}=1}^{q_{2}} \frac{\varphi_{B}\left(k_{i_{i 2}}^{(2)}\right)}{\varphi_{B}\left(k_{i_{1}}^{(2)}\right)}
$$

(b) Inherited negative binomial distributional pattern $(N B \times N B)$ (c. f. (4.9))

$$
E_{N B \times N B}^{*}\left\{\phi_{2}^{[j]}[A]\right\}=\sum_{i_{1}=1}^{q_{1}} \frac{\varphi_{N B}\left(k_{i 1}^{(1)}\right)}{\varphi_{N B}\left(k^{(1)}\right)} \sum_{i_{2}=1}^{q_{2}} \frac{\varphi_{N B}\left(k_{i_{12}}^{(2)}\right)}{\varphi_{N B}\left(k_{i_{1}}^{(2)}\right)} .
$$

(c) Inherited Poisson distributional pattern $(P \times P)$ (c. f. (4.13))

$$
E_{P \times \boldsymbol{P}}^{*}\left\{\phi_{2}^{[j]}[A]\right\}=\sum_{i_{1}=1}^{q_{1}} \frac{\varphi_{P}\left(k_{i 1}^{(1)}\right)}{\varphi_{P}\left(k^{(1)}\right)} \sum_{i_{2}=1}^{q_{2}} \frac{\varphi_{P}\left(k_{i_{i 2}}^{(2)}\right)}{\varphi_{P}\left(k_{i_{1}}^{(2)}\right)} .
$$

Example 5.3. Three stage homogeneous distributional pattern. This is characterized by three systems of probability distribution elements $g^{(i)} d \mu^{(i)}(i=1,2,3)$, which may and will be denoted by $G d \mu^{(1)} \times S d \mu^{(2)} \times L d \mu^{(3)}$ in a symbolic way, abbreviated by $G \times S \times L$, where $G$ (global), $S G$ (or $S$ ) (subglobal) and $L$ (local) corresponds to $g^{(1)}, g^{(2)}$ and $g^{(3)}$ respectively.

$$
E_{G \times S \times L}^{*}\left\{\phi_{s}[A]\right\}=\sum_{i_{1}=1}^{q_{1}} \frac{\varphi_{G}\left(k_{i 1}^{(1)}\right)}{\varphi_{G}\left(k^{(1)}\right)} \sum_{i_{2}=1}^{q_{2}} \frac{\varphi_{S}\left(k_{i_{1} i_{2}}^{(2)}\right)}{\varphi_{S}\left(k_{i_{1}}^{(2)}\right)} \sum_{i_{3}=1}^{q_{3}} \frac{\varphi_{L}\left(k_{i_{1 i z i 3}}^{(3)}\right)}{\varphi_{L}\left(k_{i_{1 i 2}}^{(3)}\right)},
$$

which turns out to

$$
E_{G \times S \times L}^{*}\left\{\phi_{3}[A]\right\}=\frac{1}{\varphi_{L}\left(k^{(3)}\right)} \sum_{i_{1}=1}^{q_{1}} \sum_{i_{2}=1}^{q_{2}} \sum_{i_{3}=1}^{q_{3}} \varphi_{L}\left(k_{i_{1 i} i_{3}}^{(3)}\right),
$$

particularly when three-stage distributional pattern is inherited in the 3-depth, because $\varphi_{G}(k)=\varphi_{S}(k)=\varphi_{L}(k)$ for every $k$ in the common domain of definition and $k_{i_{1} i_{2} \cdots i_{j-1}}^{(j)}$ $=k_{i_{1} i_{2} \cdots i_{j}}^{(j-1)}$. 


\section{$\S 6$. Partitional pattern of additive parameters in decomposition system $D^{(m)}(A)$.}

We prepare

Definition 8. A set of additive parameters $\left\{k_{i_{1} i_{2} \cdots i_{2}}^{(\nu)}\right\}(\nu=h+1, h+2, \cdots, h-d)$ in the decomposition system $D^{(m)}(A)$ is said to be equipartitioned at the d-depth in the set $A_{i_{1} i_{2} \cdots i_{h}}$ when there hold equalities

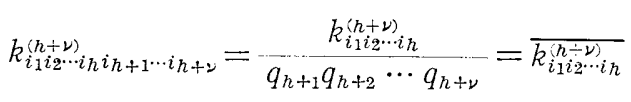

for $i_{j}=1,2, \cdots, q_{j} ; j=h+1, h+2, \cdots, h+\nu ; \nu=1,2, \cdots, d$.

Definition 9. A set of additive parameters $\left\{k_{i_{1} i_{2} \cdots i_{\nu}}^{(\nu)}(\nu=h+1, h+2, \cdots, h+d)\right.$ in the decomposition system $D^{(m)}(A)$ is said to be monopolistically concentrated in the set $A_{i_{1} i_{2} \cdots i_{h}}$ when there exists a sequence of $d$ integers $\left(i_{h+1}^{0}, i_{h+2}^{0}, \cdots, i_{h+d}^{0}\right)$ such that

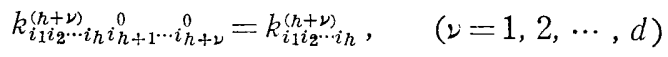

and hence

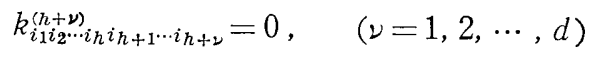

for every $\left(i_{h+1}, i_{h+2}, \cdots, i_{h+\nu}\right) \neq\left(i_{h_{1}+1}^{0}, i_{h_{1}+2}^{0}, \cdots, i_{h+\nu}^{0}\right)$.

We observe

Proposition 7. Let a set of additive parameters $\left\{k_{i_{1} i_{2} \cdots i_{\nu}}^{(\nu)}(\nu=h+1, h+2, \cdots, h+d)\right.$ in the decomposition system $D^{(m)}(A)$ be equipartitioned at the d-depth in the set $A_{i_{1} i_{2} \cdots i_{h}}$. Then we have

$$
\begin{aligned}
& E^{*}\left\{\phi_{d}\left[A_{i_{1} i_{2} \cdots i_{h}}\right]\right\}=\sum_{i_{h+1}=1}^{q_{h+1}} \frac{q_{h+1} \varphi_{1_{1} \cdots i_{h}}^{(h+1)}\left(\overline{k_{i 12}^{(h+1)} \cdots i_{h}}\right)}{\varphi_{i_{12} \cdots i_{h}}^{(h+1)}\left(k_{i_{1 i 2} \cdots i_{h}}^{(h+1)}\right)} .
\end{aligned}
$$

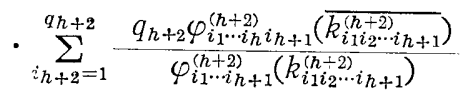

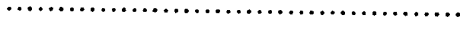

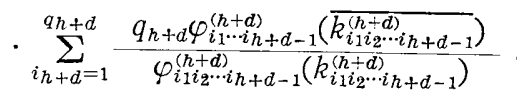

In particular when the set of $q_{h_{1}} \cdots q_{h_{2}}$ stochastic variables $\left\{X_{i_{1} \cdots i_{h} \cdots i_{h+1} \cdots i_{h+d}}\right\}\left(i_{j}=1\right.$, $\left.2, \cdots, q_{j} ; j=h+1, \cdots, h+d\right)$ is both homogeneous and inherited with the $d$-depth to the h-th distributional pattern in the set $A_{i_{1} i_{2} \cdots i_{h}}$, then we have

$$
E *\left\{\phi_{d}\left[A_{i_{1} i_{2} \cdots i_{h}}\right]\right\}=\frac{q_{h+1} \cdots q_{h+d} \varphi_{i 1 i^{\prime} \cdots i_{h}}^{(h+1)}\left(\overline{k_{i 1 i_{2} \cdots i_{h}}^{(h+d)}}\right)}{\varphi_{i_{1 i 2} \cdots i_{h}}^{(h+1)}\left(k_{i 1 i 2 \cdots \cdots}^{(h+d)}\right)} .
$$

Proposition 8. Let a set of additive parameters $\left\{k_{i 1 i 2 \cdots i_{\nu}}^{(\nu)}\right\}(\nu=h+1, h+2, \cdots, h+d)$ in the decomposition system $D^{(m)}(A)$ be monopolistically concentrated in $A_{i_{1} i_{2} \cdots i_{h}}$ at the d-depth. Then we have

$$
E^{*}\left\{\phi_{d}\left[A_{i_{1} i_{2} \cdots i_{h}}\right]\right\}=1 .
$$

\section{$\S 7$. $\phi$-dispersion as function of size variable.}

Let us consider a sequence of $\phi$-dispersion $\left\{\phi_{d}[A]\right\}(d=1,2, \cdots, m)$ for an assigned decomposition system $D^{(m)}(A)$ of an assigned set $A$. It is now our chief concern how 
$\phi_{d}[A]$ will change as a function of the depth $d$. In discussing such problem there are two fundamental tools which we propose to use.

$\left(1^{\circ}\right)$ Size variable associated with the decomposition system $D^{(m)}(A)$. Let us assume every subset $A_{i_{1} i_{2} \cdots i_{m}}$ is measurable, and let its measure be denoted by $m\left(A_{i_{1} i_{2} \cdots i_{m}}\right)$. Let us denote by $a_{h}$ the average of all $\left\{m\left(A_{i_{1} i_{2} \cdots i_{h}}\right)\right\}\left(i_{j}=1,2, \cdots, q_{i} ; j=1,2, \cdots, h\right.$, $h=1,2, \cdots, m)$.

$$
a_{h}=\frac{1}{q_{1} q_{2} \cdots q_{h}}-\sum_{i_{1}=1}^{q_{1}} \cdots \sum_{i_{h}=1}^{q_{h}} m\left(A_{i_{1} i_{2} \cdots i_{h}}\right),
$$

which is called the $h$-th average size associated with $D^{(h)}(A)$.

For the sake of convenienience we add to define

$$
a_{0}=A \text {. }
$$

Under these preparations we introduce

Definition 10. A sequence of functions $\left\{\Phi\left(a_{i} \mid A\right)\right\}(i=0,1,2, \cdots, m)$ defined by

$$
\begin{aligned}
& \Phi\left(a_{0} \mid A\right)=1, \\
& \Phi\left(a_{1} \mid A\right)=\sum_{i_{1}=1}^{q_{1}} \frac{\varphi^{(1)}\left(k_{i 1}^{(1)}\right)}{\varphi^{(1)}\left(k^{(1)}\right)}, \\
& \Phi\left(a_{h} \mid A\right)=\sum_{i_{1}=1}^{q_{1}} \frac{\varphi^{(1)}\left(k_{i 1}^{(1)}\right)}{\varphi^{(1)}\left(k^{(1)}\right)} \sum_{i_{2}=1}^{q_{2}} \frac{\varphi_{i 1}^{(2)}\left(k_{i 12}^{(2)}\right)}{\varphi_{i_{1}}^{(2)}\left(k_{i 1}^{(2)}\right)} \cdots \sum_{i_{h}=1}^{q_{h}} \frac{\varphi_{i_{1} \cdots i_{h-1}}^{(h)}\left(k_{i 12}^{(h)} \cdots i_{h}\right)}{\varphi_{i 1}^{(h) \cdots i_{h-1}}\left(k_{i 1 i_{2} \cdots i_{h-1}}^{(h)}\right)}
\end{aligned}
$$

for $h=2,3, \cdots, m$ is said to constitute a $\phi$-dispersion system as a function of the: average size $a_{h}(h=0,1,2, \cdots, m)$.

Particularly when all $A_{i_{1} i_{2} \cdots i_{h}}$ have the same measure, i.e.,

$$
m\left(A_{i_{1} i_{2} \cdots i_{h}}\right)=\frac{m(A)}{q_{1} q_{2} \cdots q_{h}}=a_{h}
$$

for $i_{j}=1,2, \cdots, q_{j} ; j=1,2, \cdots, h ; h=1,2, \cdots, m$, each $a_{h}$ is the size of each subset in the h-th stage decomposition.

In view of these facts we may and we shall call $a_{h}$ to be size variable which is monotone decreasing: $a_{0}>a_{1}>\cdots>a_{m}$.

$\left(2^{\circ}\right)$ Homogeneous and inherited multistage distributional pattern interpolated between the $h$-th stage decomposition $D_{h}^{(m)}(A)$ and the $(h+1)$-th stage one $D_{h+1}^{(m)}(A)$. To explain the principal aspects of our techniques, let us consider the simplest $\phi$-dispersion

$$
\phi_{1}[A]=\Phi\left(a_{1} \mid A\right)=\frac{1}{\varphi^{(1)}\left(k^{(1)}\right)} \sum_{i_{1}=1}^{q_{1}} \varphi^{(1)}\left(k_{i_{1}}^{(1)}\right) .
$$

Now let us consider the case when the non-negative integer $q_{1}$ is decomposed into the product of $l_{1}$ non-negative integers $\left\{q_{1 \nu}\right\}\left(\nu=1,2, \cdots, l_{1}\right)$ such that $q_{1}=q_{11} q_{12} \cdots q_{1 l_{1}}$. Then it is possible to establish one-to-one correspondence defined by $i=\phi\left(i_{1}, i_{2}, \cdots, i_{l}\right)$ where the variable (integer) $i$ ranges from 1 to $q_{1}$ while $i_{j}=1,2, \cdots, q_{1 j} ; j=1,2, \cdots, l_{1}$. In this connection we define $A_{i_{11} i_{2} \cdots i_{l}}^{(1)}=A_{\psi\left(i_{1}, i_{2}, \cdots, i_{1}\right)}$ and $k_{i_{1} i_{2} \cdots i_{1}}^{(1)}=k_{\psi\left(i_{1}, i_{2}, \cdots, i_{1}\right)}^{(1)}$.

In the consequence we may rewrite (7.7) as

$$
\phi_{1}[A]=\frac{1}{\varphi^{(1)}\left(\bar{k}^{(1)}\right)} \sum_{i_{1}=1}^{q_{11}} \sum_{i_{2}=1}^{q_{12}} \cdots \sum_{i_{l_{1}=1}=1}^{q_{111}} \varphi^{(1)}\left(k_{i_{1 i 2} \cdots i l_{1}}^{(1)}\right),
$$


which can be reformed as

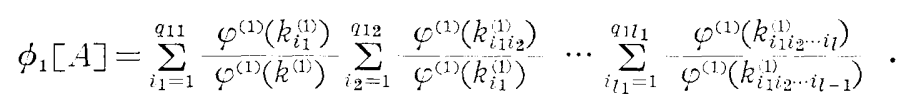

The expression (7.9) of $\dot{\phi}_{1}[A]$ shows that the 1 -depth $\dot{\phi}$-dispersion can be interpretated as the $l_{1}$-depth $\phi$-dispersion which is homogeneous and inherited in the $l_{1}$-depth in the set $A$ in the sense of Definitions 4 and 5 .

In view of this fact let us now introduce a sequence of area sizes $\left\{a_{\nu}^{(1)}\right\}(\nu=1,2$, $\cdots, l_{1}$ ) defined by

$$
a_{\nu}^{(1)}=\frac{1}{q_{11}}-\frac{1}{q_{12}} \cdots q_{q_{1 \nu}}^{q_{11}} \sum_{i_{1}=1} \cdots \sum_{i_{\nu}=1}^{q_{1}} m\left(\mathcal{A}_{i 1 i_{2} \cdots i_{\nu}}^{(1)}\right)
$$

and, with reference to this sequence, let us define a sequence of $\dot{\phi}$-dispersions

for $\nu=1,2, \cdots, l_{1}$.

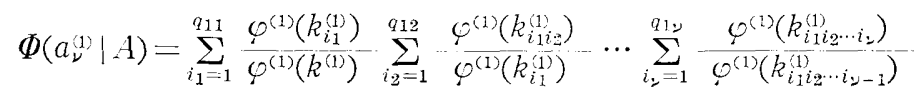

$$
\begin{aligned}
& =\frac{1}{\varphi^{(1)}\left(k^{(1)}\right)} \sum_{i_{1}=1}^{q_{11}} \cdots \sum_{i_{\nu}=1}^{q_{\nu 1}} \varphi^{(i)}\left(k_{i_{1}\left(12 \cdots i_{2}\right.}^{(1)}\right)
\end{aligned}
$$

It is noted that $a_{0}>a_{1}^{(1)}>a_{2}^{(1)}>\cdots>a_{1-1}^{(1)}>a_{1}^{(1)}=a_{1}$ and that the sequence $\left\{\Phi\left(a_{\nu}^{(1)} \mid A\right)\right\}$ $\left(\nu=1,2, \cdots, l_{1}\right)$ is an interpolation between $\Phi\left(a_{0} \mid A\right)$ and $\Phi\left(a_{1} \mid A\right)$.

The interpolation technique just now explained with respect to the first stage decompostion $D_{1}^{(m)}(A)$ and the second stage one $D_{2}^{(m)}(A)$ can be similarly defined in the connection with the $h$-th etage $D_{h}^{(m)}(A)$ and the $(h+1)$-th stage $D_{h+1}^{(m)}(A)$ for $h=2,3$, $\cdots, m-1$. In this way it may be possible to introduce a sequence of the average sizes $\left\{a_{\nu}^{(h)}\right\}\left(\nu=1,2, \cdots, l_{\nu} ; \nu=1,2, \cdots, m\right)$ such that

$$
a_{\nu-1}>a_{1}^{(\nu)}>a_{2}^{(\nu)}>\cdots>a_{l_{\nu}}^{(\nu)}=a_{\nu}
$$

and to define a sequence of $\dot{\phi}$-dispersions

$$
\Phi\left(a_{s}^{(\nu)} \mid A\right)
$$

for $s=1,2, \cdots, l_{\nu} ; \nu=1,2, \cdots, m$, as a whole. In this manner we can have a graph

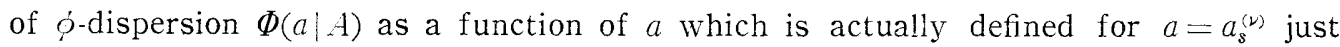
mentioned.

$\left(3^{\circ}\right)$ Equipartition of additive parameters in the homogeneous and inherited multistage distributional pattern in the decomposition system $D^{(m)}(A)$ with $\left\{A_{i_{1} i_{2} \cdots i_{m}}\right\}$ of equal measure $m\left(A_{i_{1} i_{2} \cdots i_{m}}\right)=m(A) /\left(q_{1} q_{2} \cdots q_{m}\right)$.

In this case we have

$$
k_{i 12 \cdots i h}^{h>}=k_{i 12 ; \cdots i_{h}}^{(m)}=K / q_{1} q_{2} \cdots q_{h}=K a_{h} a_{0}^{-1}
$$

and

by putting

$$
\Phi\left(a_{h} \mid A\right)=\frac{a_{h}^{-1} \varphi\left(K a_{h} a_{0}^{-1}\right)}{a_{0}^{-1} \varphi(K)}=\frac{\varphi\left(b_{h} K\right)}{b_{h} \varphi(K)}, \quad \text { say }
$$

$$
b_{12}=a_{i 2} a_{10}^{-1}
$$

which is called to be a relative area variable, or an area-index. A relative area variable is convenient when the set $A$ is assigned, because in this scale the relative 
area is equal to 1 for the set $A$.

A deep insight into the structure of multistage distributional pattern can be obtained by use of the $\phi$-dispersion function $\Phi(a \mid A)$ as a function of size $a$ in the context just explained.

It is noted that the behaviours of the $\phi$-dispersion function $\Phi(a \mid A)$ for each of nine two stage and twenty seven three stage distributional patterns given in Examples 3.4 and 3.5 are worth while to particular numerical investigations. Indeed numerical investigations based upon our theoretical formulation can yield us some of the possible reasons to the experimental results on natural and artificial populations which have been given by Morishita [2].

\section{$\S 8$. Index functions of dispersion as a function of size variable.}

In connection with current uses of index of dispersion, it is useful to introduce

Definition 11. (1 $\left.{ }^{\circ}\right) I_{\bar{d}^{j}}\left[A_{i_{1} i_{2} \cdots i_{h}}\right]$ defined in (2.11) is said to be the d-depth index of dispersion of the $j$-th order in the set $A_{i_{1}, i_{2}, \cdots, i_{h}}$.

$\left(2^{\circ}\right)$ A sequence of functions $I^{-j}\left(a_{i} \mid A\right)(i=0,1,2, \cdots, m)$ defined by

$$
\begin{aligned}
& I^{[j]}\left(a_{0} \mid A\right)=\left(q_{1} q_{2} \cdots q_{m}\right)^{j-1}, \\
& I^{[j]}\left(a_{\nu} \mid A\right)=E^{*}\left\{I_{\nu}^{[j]}[A]\right\},
\end{aligned}
$$

for $\nu=1,2, \cdots, m$, is said to be an index function of dispersion of the $j$-th order as $a$ function of size variable which takes the values $\left\{a_{\nu}\right\}(\nu=0,1,2, \cdots, m)$.

The current index of dispersion used and advocated by Morishita [2] [5] and others is the 1-depth index of dispersion of the second order in our terminology. Apart from a multiplier $\left(q_{1} q_{2} \cdots q_{n}\right)^{j-1}$, the essential aspects of $I_{d}^{[j]}[A]$ can be observed from our general considerations on $\phi_{d}[A]$ and those of $I^{[j]}\left(a_{i} \mid A\right)$ from those of functions $\Phi\left(a_{i} \mid A\right)$. We do not think the multiplier is so essential in our general approach. The sole merits, so far as the results of the present paper are concerned, can be recognised from the fact that we have

$$
E_{P}^{*}\left\{I_{d}^{[j]}[A]\right\}=1
$$

for a homogeneous and inherited multistage distributional patterns based upon Poisson distribution. Multipliers are due to partly to the tradition and partly to the fact that theoretical emphasis may be placed upon Poisson distribution, as we can observe from

$$
E^{*}\left\{I_{d}^{[j]}[A]\right\}=1
$$

for a homogeneous and inherited multistage distributional based upon Poisson distribution.

Kyushu University

\section{Literature}

[1: Kitagawa, T., Successive process of statistical inference associated with an additive family of sufficient statistics, Bull. Math. Stat., 7, No. 3-4 (1957), 92-112.

[2] Morishita, M., Measuring of the dispersion of individuals and analysis of the distributional pattern, Mem. Fac. Sci. Kyushu Univ., Ser. E (Biol.), 2 (1957), 215-235. 
[3] Morishita, M., $I_{0}$-index, a measure of dispersion of individuals, Res. Popul. Ecol., 4 (1962), $1-7$.

[4] Morishita, M., Application of $I_{j}$-index to sampling techniques, Res. Popul. Ecol., 6 (1964), 43-53.

[5] Morishita, M., A revision of the methods for estimating population values of the index of dispersion in the $I_{\overline{0}}$-method, Res. Popul. Ecol., 7 (1965), 126-128.

[6] Smith, E.H., Measurement of diversity, Nature, 163 (1949), 688. 